\title{
From a Cerium-Doped Polynuclear Bismuth Oxido Cluster to $\beta-\mathrm{Bi}_{2} \mathrm{O}_{3}: \mathrm{Ce}$
}

\author{
Marcus Weber, ${ }^{\text {a,e }}$ Tobias Rüffer, ${ }^{b}$ Florian Speck, ${ }^{c, e}$ Fabian Göhler, ${ }^{c, e}$ Dominik P. Weimann, ${ }^{\text {d }}$ Christoph A. \\ Schalley, Thomas Seyller, ${ }^{c, e}$ Heinrich Lang, ${ }^{b, e}$ Michael Mehring, ${ }^{\mathrm{a}, *, e}$ \\ ${ }^{a}$ Fakultät für Naturwissenschaften, Institut für Chemie, Professur Koordinationschemie, Technische Universität Chemnitz, \\ 09111 Chemnitz (Germany) \\ ${ }^{b}$ Fakultät für Naturwissenschaften, Institut für Chemie, Professur Anorganische Chemie, Technische Universität Chemnitz, \\ 09111 Chemnitz (Germany) \\ 'Fakultät für Naturwissenschaften, Institut für Physik, Professur für Experimentalphysik mit dem Schwerpunkt Technische \\ Physik, Technische Universität Chemnitz, 09126 Chemnitz (Germany) \\ ${ }^{\mathrm{d}}$ Fachbereich Biologie, Chemie, Pharmazie, Institut für Chemie und Biochemie, Professur Organische Chemie, Freie \\ Universität Berlin, 14195 Berlin (Germany) \\ ${ }^{\mathrm{e}}$ Center for Materials, Architectures and Integration of Nanomembranes (MAIN), 09126 Chemnitz (Germany)
}

KEYWORDS Bismuth Oxido Cluster, Rare Earth, Doping, Bismuth(III) Oxide, Polymorphism, Photocatalysis.

\section{ELECTRONIC SUPPLEMENTARY INFORMATION}

\section{Crystallographic Characterization of $\left[\mathrm{Bi}_{38} \mathrm{O}_{45}\left(\mathrm{NO}_{3}\right)_{24}(\mathrm{DMSO})_{28}\right]: \mathrm{Ce} \cdot 1.5 \mathrm{DMSO}(1)$ and $\mathrm{Ce}\left(\mathrm{NO}_{3}\right)_{3} \cdot 4 \mathrm{DMSO}(5)$}

The single crystals of $\mathbf{1}$ and $\mathbf{5}$ were selected and mounted in Krytox ${ }^{\oplus}$. The low stability of the single crystals of $\mathbf{1}$ in Krytox ${ }^{\oplus}$ leads to a rapid loss of the DMSO as packing solvent, resulting in a lower DMSO content in the X-ray diffraction analysis compared to the results from CHNS-analysis.

In case of $\left[\mathrm{Bi}_{38} \mathrm{O}_{45}\left(\mathrm{NO}_{3}\right)_{24}(\mathrm{DMSO})_{28}\right]: \mathrm{Ce} \cdot 1.5 \mathrm{DMSO}(\mathbf{1})$, the determined unit cell did already indicate that the $\mathrm{Bi}_{38} \mathrm{O}_{45}-\mathrm{oxido}$ cluster related to $\left[\mathrm{Bi}_{38} \mathrm{O}_{45}\left(\mathrm{NO}_{3}\right)_{20}(\mathrm{DMSO})_{28}\right]\left(\mathrm{NO}_{3}\right)_{4} \cdot 4 \mathrm{DMSO}(\mathbf{A})^{1}$ was formed. We took now the final ins file of $\mathbf{A}$ and let it refine against the newly determined .hkl files of $\mathbf{1}$. That worked already very well. Next, we used the EADP and EXYZ instruction and allowed a free refinement including further FVAR parameter of the Bi and Ce content on the chosen Bi atom. A typical example is given next, here for the $\mathbf{1}$ case:

$\begin{array}{cccccccc}\text { BI5 } & 5 & 0.192353 & 0.722575 & 0.643727 & 21.00000 & 0.03624 & 0.02090= \\ & 0.01555 & -0.00073 & 0.01367 & -0.00435 & & \\ \text { CE5 } & 7 & 0.192353 & 0.722575 & 0.643727 & -21.00000 & 0.03624 & 0.02090= \\ & 0.01555 & -0.00073 & 0.01367 & -0.00435 & & \end{array}$

We checked for each individual Bi atom of 1 whether the obtained FVAR parameter $(21.0 /-21.0)$ in the case given above deviate from 1.000. In case of obtained FVAR parameters $>1.000$ we deleted any further check of a potential Ce content and set the occupation of this $\mathrm{Bi}$ atom to 11.000 , thus to $100 \%$. Only in cases where the obtained FVAR parameter went below 0.995 we kept the respective occupation disorder. Then we observed the following occupation factors for $\mathbf{1}$ (Table S1) after final refinement (see below Notice IV) in a stepwise approach.

Notice I: In all other cases we did not observe Bi contents below 0.995.

Notice II: For 1 a certain respective Ce-content was observed/refined. As these Ce atoms correspond to Ce $\mathrm{e}^{4+}$ ions, $\mathrm{cf}$. accompanying manuscript, the number of required counter anions must be modified. For a full $\mathrm{Bi}_{38} \mathrm{O}_{45}$ cluster, like 
$\left[\mathrm{Bi}_{38} \mathrm{O}_{45}\left(\mathrm{NO}_{3}\right)_{20}(\mathrm{DMSO})_{28}\right]\left(\mathrm{NO}_{3}\right)_{4} \cdot 4 \mathrm{DMSO}(\mathbf{A}),{ }^{1}$ our preliminary work did thus establish the presence of $24 \mathrm{NO}_{3}{ }^{-}$anions. This additional content of anions could not be refined reliable (see below Notice IV) for $\mathbf{1}$ and thus, the chemical formulas remained unchanged in this respect.

There are indeed remaining unrefined electron density peaks of 5.7 and $-4.7 \mathrm{e} / \AA^{-3}$ in close proximity to a coordinating (and disordered) DMSO molecule, namely with the atoms S9, O32, C17 and C18. Likely, these peaks are due to the excess of $\mathrm{NO}_{3}{ }^{-}$ anions in $\mathbf{1}$. However, all trials to refine a partially occupied $\mathrm{NO}_{3}{ }^{-}$anion and a disordered DMSO molecule gave non-reliable results.

Notice III: At that stage of the refinement we took the .ins file $\mathbf{1}$ and refined it against the .hkl files of $\mathbf{A}^{1}$ The thus obtained occupation factors for the $\mathrm{Bi}$ atoms under question are summarized in Table $\mathrm{S} 1$ as well, allowing a direct comparison.

Table S1. FVAR Values for Freely Refined Bi vs. Ce Atomic Site Occupation.

\begin{tabular}{|c|c|c|c|c|}
\hline \multirow[b]{2}{*}{ Bi atom } & \multicolumn{2}{|c|}{$\mathbf{1}^{\text {a) }}$} & \multicolumn{2}{|c|}{$\mathbf{A}^{1}$} \\
\hline & value & esd & value & esd \\
\hline $\mathrm{Bi} 5$ & 0.97342 & 0.00881 & 1.00883 & 0.01034 \\
\hline $\mathrm{Bi} 9$ & 0.98086 & 0.00940 & 1.01021 & 0.01099 \\
\hline Bil1 & 0.96924 & 0.00920 & 1.00906 & 0.01062 \\
\hline Bi15 & 0.93434 & 0.00866 & 1.00075 & 0.01001 \\
\hline Bi:Ce ratio & \multicolumn{2}{|c|}{$\mathrm{Bi}_{75.44} \mathrm{Ce}_{0.56}$} & \multicolumn{2}{|c|}{$\mathrm{Bi}_{76.10} \mathrm{Ce}_{0}$} \\
\hline
\end{tabular}

a) This work.

Notice IV: For further refinement of $\mathbf{1}$ an additional number of restraints, if compared to $\mathbf{A}$, was required. Likely, this might be caused by the presence of more than $24 \mathrm{NO}_{3}{ }^{-}$anions, cf. above, for example on positions where DMSO solvent molecules have been refined before. Furthermore, in case of both $\mathbf{1}$ the final checkcif procedure revealed large VOIDS. As no further DMSO packing solvents could be refined reliable, SQUEEZE was used to treat the data set. The solvent accessible volume within the unit cell (SAV) and the electrons found by SQUEEZE within the SAV amount to: $1=1186.1 \AA$ and 424.7 e. The values correspond well with the presence of up to ten further DMSO packing solvents (electron count $=420$ ) in the unit cell. It is verified further that by applying SQUEEZE the occupation factors given in Table S1 were not affected. 


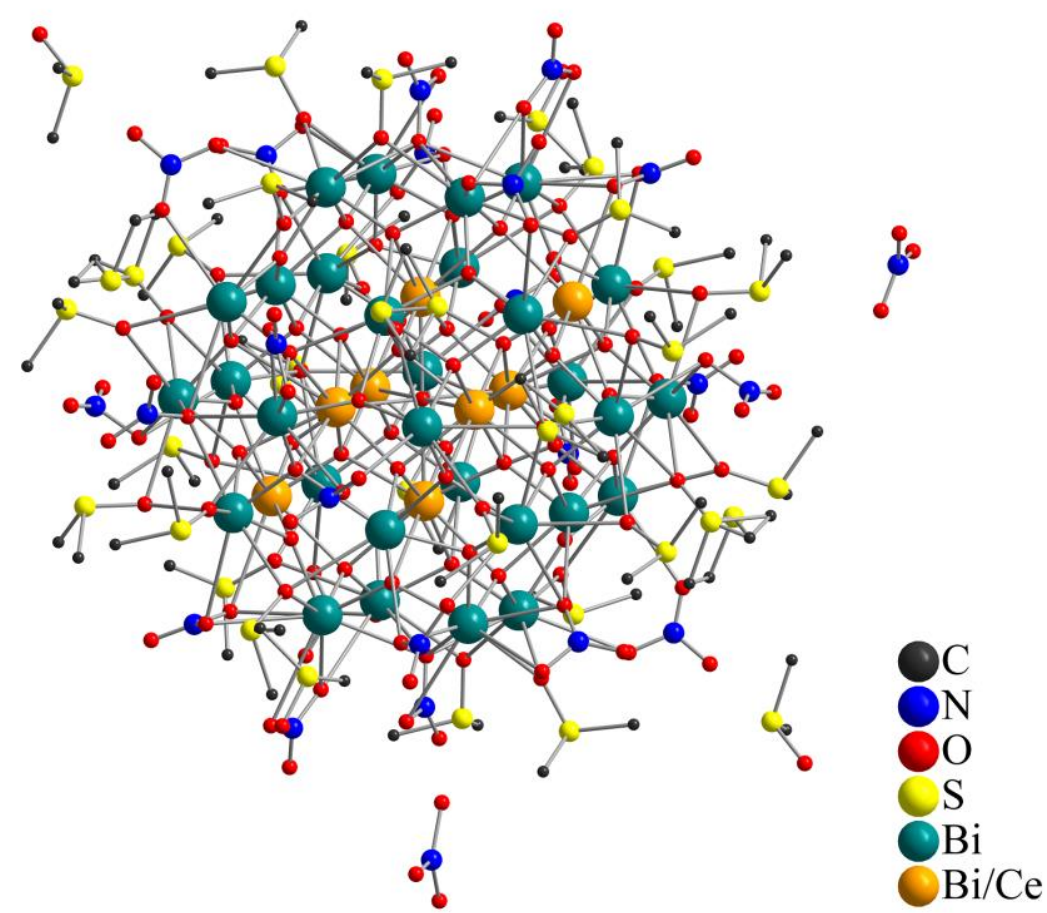

Figure S1. "Ball-and-stick" model of the bismuth oxido cluster $\left[\mathrm{Bi}_{38} \mathrm{O}_{45}\left(\mathrm{NO}_{3}\right)_{24}(\mathrm{DMSO})_{28}\right]$ :Ce.1.5DMSO (1). Hydrogen atoms are omitted for clarity.

Table S2. Assignment of the Binding Energies Based on the XPS Spectra of the Bismuth Oxido Clusters $\left[\mathrm{Bi}_{38} \mathrm{O}_{45}\left(\mathrm{NO}_{3}\right)_{24}(\mathrm{DMSO})_{28}\right]: \mathrm{Ce} \cdot 1.5 \mathrm{DMSO}(1, \mathrm{Ce}-\mathrm{content}=\mathbf{0 . 6 9 \%})$, and $\left[\mathrm{Bi}_{38} \mathrm{O}_{45}\left(\mathrm{NO}_{3}\right)_{24}(\mathrm{DMSO})_{32}\right]: \mathrm{Ce}(2$, Cecontent $=0.31 \%){ }^{2}$

\begin{tabular}{ccc}
\hline Signal & $\mathbf{1}$ & Binding Energy / eV \\
& 18.1 & $\mathbf{2}$ \\
\hline In $4 \mathrm{~d}$ & 26.9 & 18.1 \\
$\mathrm{Bi} 5 \mathrm{~d}_{5 / 2}$ & 30.0 & 26.9 \\
$\mathrm{Bi} 5 \mathrm{~d}_{3 / 2}$ & 160.1 & 30.0 \\
$\mathrm{Bi} 4 \mathrm{f}_{7 / 2}$ & 165.4 & 160.2 \\
$\mathrm{Bi} 4 \mathrm{f}_{5 / 2}$ & $\sim 231.7$ & 165.5 \\
$\mathrm{~S} 2 \mathrm{~s}$ & 285.9 & $\sim 231.5$ \\
$\mathrm{C} 1 \mathrm{~s}$ & 407.4 & 285.9 \\
$\mathrm{~N} 1 \mathrm{~s}$ & $\sim 443$ & 407.5 \\
$\mathrm{In} 3 \mathrm{~d}_{5 / 2}{ }^{*}$ & $\sim 443$ & $\sim 444$ \\
$\mathrm{Bi} 4 \mathrm{~d}_{5 / 2}{ }^{*}$ & 452.4 & $\sim 444$ \\
$\mathrm{In} 3 \mathrm{~d}_{3 / 2}$ & 466.9 & 452.4 \\
$\mathrm{Bi} 4 \mathrm{~d}_{3 / 2}$ & 533.1 & 466.9 \\
$\mathrm{O} 1 \mathrm{~s}$ & $\sim 665.9$ & 533.1 \\
In $3 \mathrm{p}_{3 / 2}$ & $\sim 681.5$ & $\sim 665.9$ \\
$\mathrm{Bi} 4 \mathrm{p}_{3 / 2}$ & $\sim 703.6$ & $\sim 681.5$ \\
In $3 \mathrm{p}_{1 / 2}$ & $\sim 807.6$ & $\sim 703.6$ \\
$\mathrm{Bi} 4 \mathrm{p}_{1 / 2}$ & 940.4 & $\sim 807.6$ \\
$\mathrm{Bi} 4 \mathrm{~s}$ & & 940.3 \\
\hline
\end{tabular}


* Signals of Bi $4 \mathrm{~d}_{5 / 2}$ and In $3 \mathrm{~d}_{5 / 2}$ are overlapping at approx. $443 \mathrm{eV}$ impeding a clear assignment of corresponding binding energies.

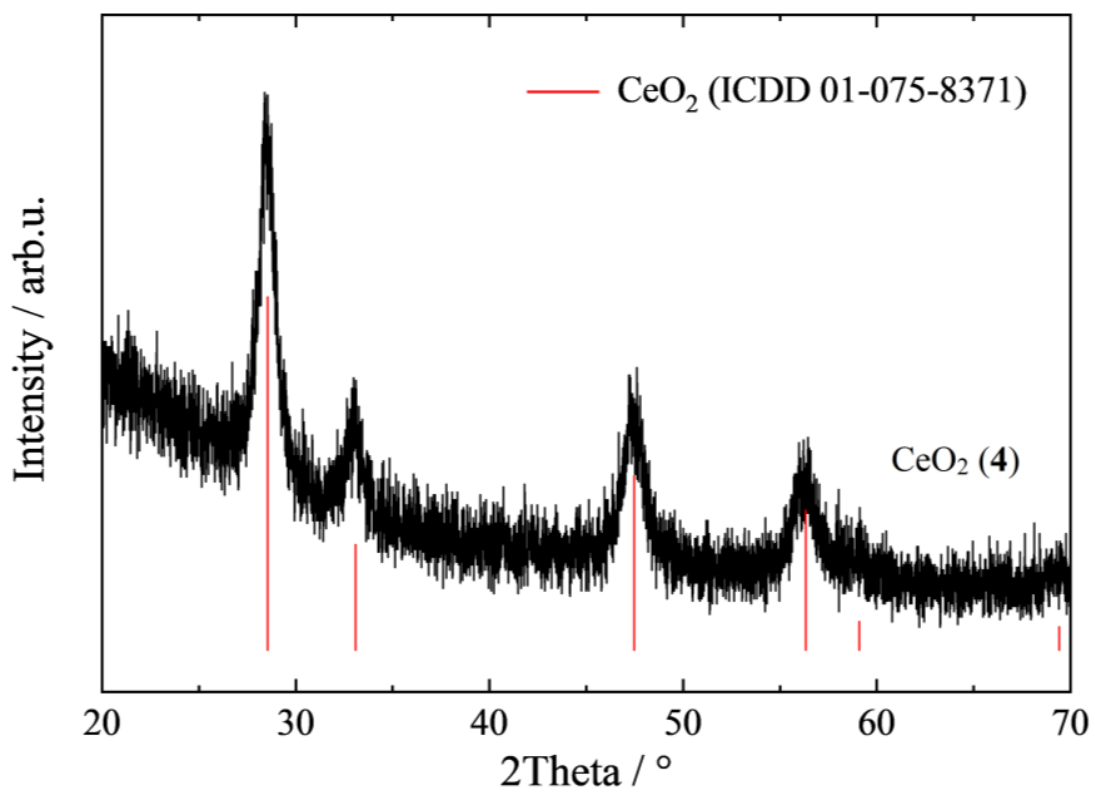

Figure S2. PXRD pattern of the as-prepared $\mathrm{CeO}_{2}(4)$ after calcination of $\mathrm{Ce}\left(\mathrm{NO}_{3}\right)_{3} \cdot 6 \mathrm{H}_{2} \mathrm{O}$ at $500{ }^{\circ} \mathrm{C}$ for $8 \mathrm{~h}$ as reference sample for XPS studies.

Table S3. Assignment of Binding Energies (eV) of the Ce 3d-region Based on the XPS Spectra of the Bismuth Oxido Clusters $\left[\mathrm{Bi}_{38} \mathrm{O}_{45}\left(\mathrm{NO}_{3}\right)_{24}(\mathrm{DMSO})_{28}\right]: \mathrm{Ce} \cdot 1.5 \mathrm{DMSO}(1, \mathrm{Ce}$-content $=0.69 \%),\left[\mathrm{Bi}_{38} \mathrm{O}_{45}\left(\mathrm{NO}_{3}\right)_{24}(\mathrm{DMSO})_{32}\right]: \mathrm{Ce}(2$, Cecontent $=0.31 \%)$, and the Internal Reference Sample $\mathrm{CeO}_{2}(4)$ Compared to Literature Reports. ${ }^{3,4}$

\begin{tabular}{ccccccc}
\hline Final state & $\begin{array}{c}\text { Spin-orbit } \\
\text { doublet }\end{array}$ & $\mathbf{1}$ & $\mathbf{2}$ & $\mathbf{4}$ & $\mathrm{CeO}_{2}{ }^{3}$ & $\mathrm{CeO}_{2}{ }^{4}$ \\
\hline $\mathrm{Ce} 3 \mathrm{~d}^{9} 4 \mathrm{f}^{0} \mathrm{O} 2 \mathrm{p}^{6}$ & $\mathrm{Ce} 3 \mathrm{~d}_{5 / 2}\left(\mathrm{v}^{\prime \prime \prime}\right)$ & 898.5 & 898.7 & 898.5 & $898.3 \pm 0.1$ & $898.1 \pm 0.1$ \\
$\mathrm{Ce} 3 \mathrm{~d}^{9} 4 \mathrm{f}^{1} \mathrm{O} 2 \mathrm{p}^{5}$ & $\mathrm{Ce} 3 \mathrm{~d}_{5 / 2}\left(\mathrm{v}^{\prime \prime}\right)$ & 888.8 & 888.5 & 888.7 & $888.5 \pm 0.1$ & $888.5 \pm 0.1$ \\
$\mathrm{Ce} 3 \mathrm{~d}^{9} 4 \mathrm{f}^{2} \mathrm{O} 2 \mathrm{p}^{4}$ & $\mathrm{Ce} 3 \mathrm{~d}_{5 / 2}(\mathrm{v})$ & 882.9 & 883.0 & 882.7 & $882.7 \pm 0.1$ & $882.6 \pm 0.1$ \\
$\mathrm{Ce} 3 \mathrm{~d}^{9} 4 \mathrm{f}^{0} \mathrm{O} 2 \mathrm{p}^{6}$ & $\mathrm{Ce} 3 \mathrm{~d}_{3 / 2}\left(\mathrm{u}^{\prime \prime \prime}\right)$ & 916.9 & 916.9 & 916.9 & $916.9 \pm 0.1$ & $916.8 \pm 0.1$ \\
$\mathrm{Ce} 3 \mathrm{~d}^{9} 4 \mathrm{f}^{1} \mathrm{O} 2 \mathrm{p}^{5}$ & $\mathrm{Ce} 3 \mathrm{~d}_{3 / 2}\left(\mathrm{u}^{\prime \prime}\right)$ & 908.0 & 907.8 & 907.5 & $907.3 \pm 0.1$ & $907.3 \pm 0.1$ \\
$\mathrm{Ce} 3 \mathrm{~d}^{9} 4 \mathrm{f}^{2} \mathrm{O} 2 \mathrm{p}^{4}$ & $\mathrm{Ce} 3 \mathrm{~d}_{3 / 2}(\mathrm{u})$ & 901.2 & 901.1 & 901.2 & $901.3 \pm 0.1$ & $901.2 \pm 0.1$ \\
\hline
\end{tabular}




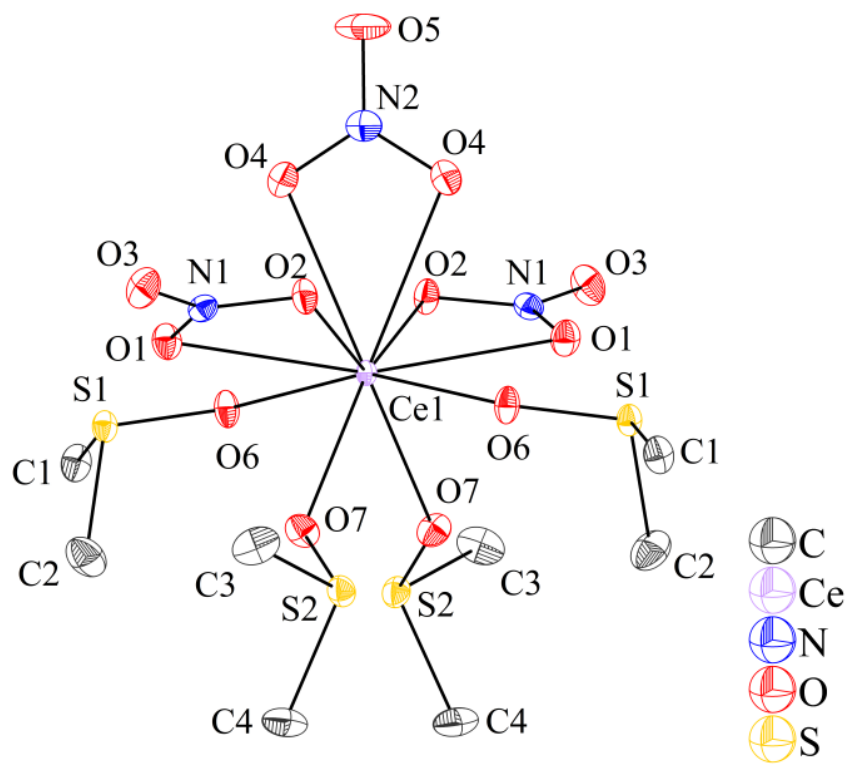

Figure S3. Molecular structure of $\mathrm{Ce}\left(\mathrm{NO}_{3}\right)_{3} \cdot 4 \mathrm{DMSO}(\mathbf{5})$ in the solid state (50\% probability level of displacement ellipsoids, hydrogen atoms are omitted for clarity).

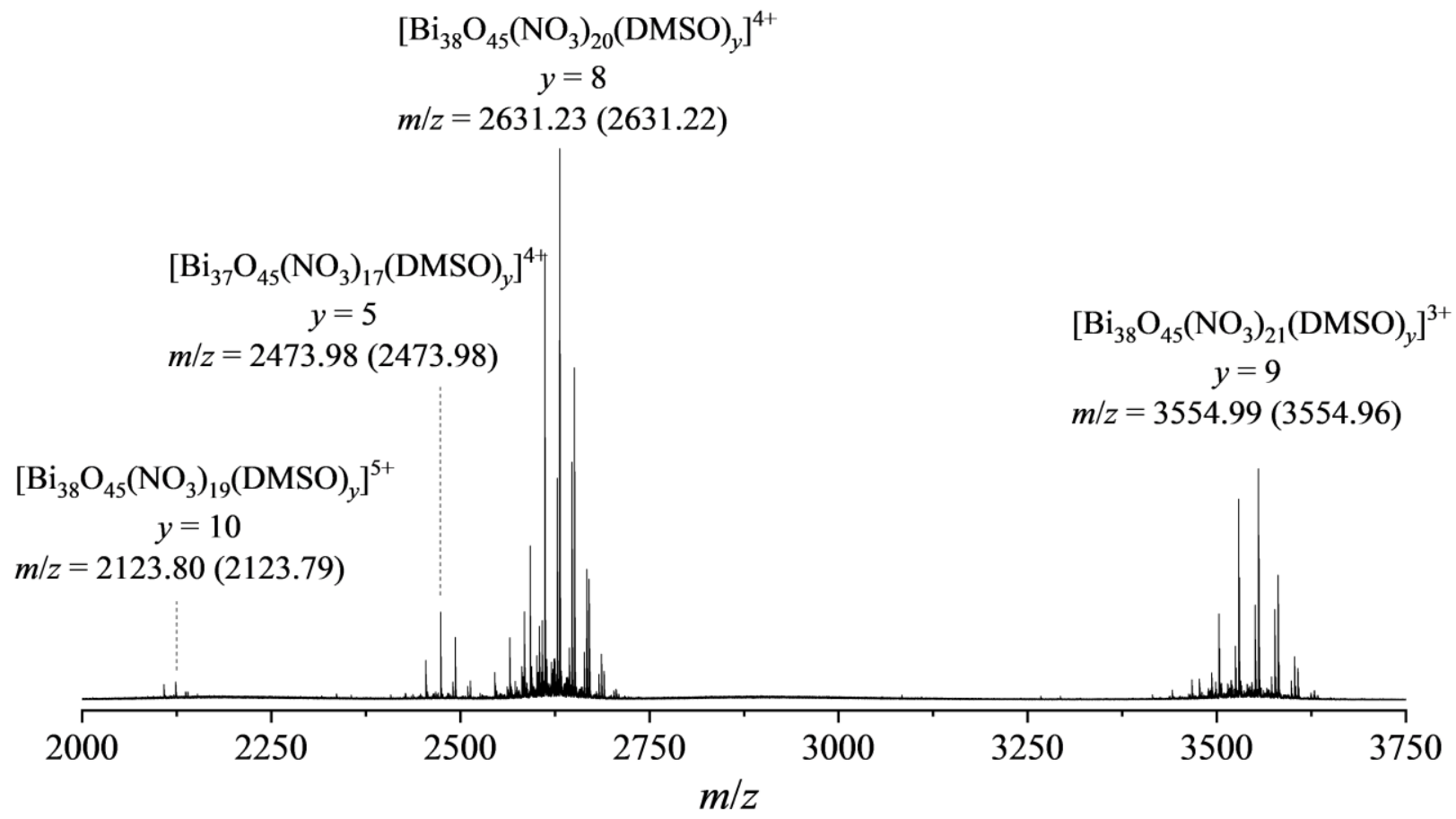

Figure S4. Survey ESI-MS spectrum of $\left[\mathrm{Bi}_{38} \mathrm{O}_{45}\left(\mathrm{NO}_{3}\right)_{24}(\mathrm{DMSO})_{28}\right]: \mathrm{Ce} \cdot 1.5 \mathrm{DMSO}(\mathbf{1})$ electrosprayed from a mixture of DMSO and $\mathrm{MeCN}(v: v=0.05: 0.95, c=100 \mu \mathrm{M})$. 


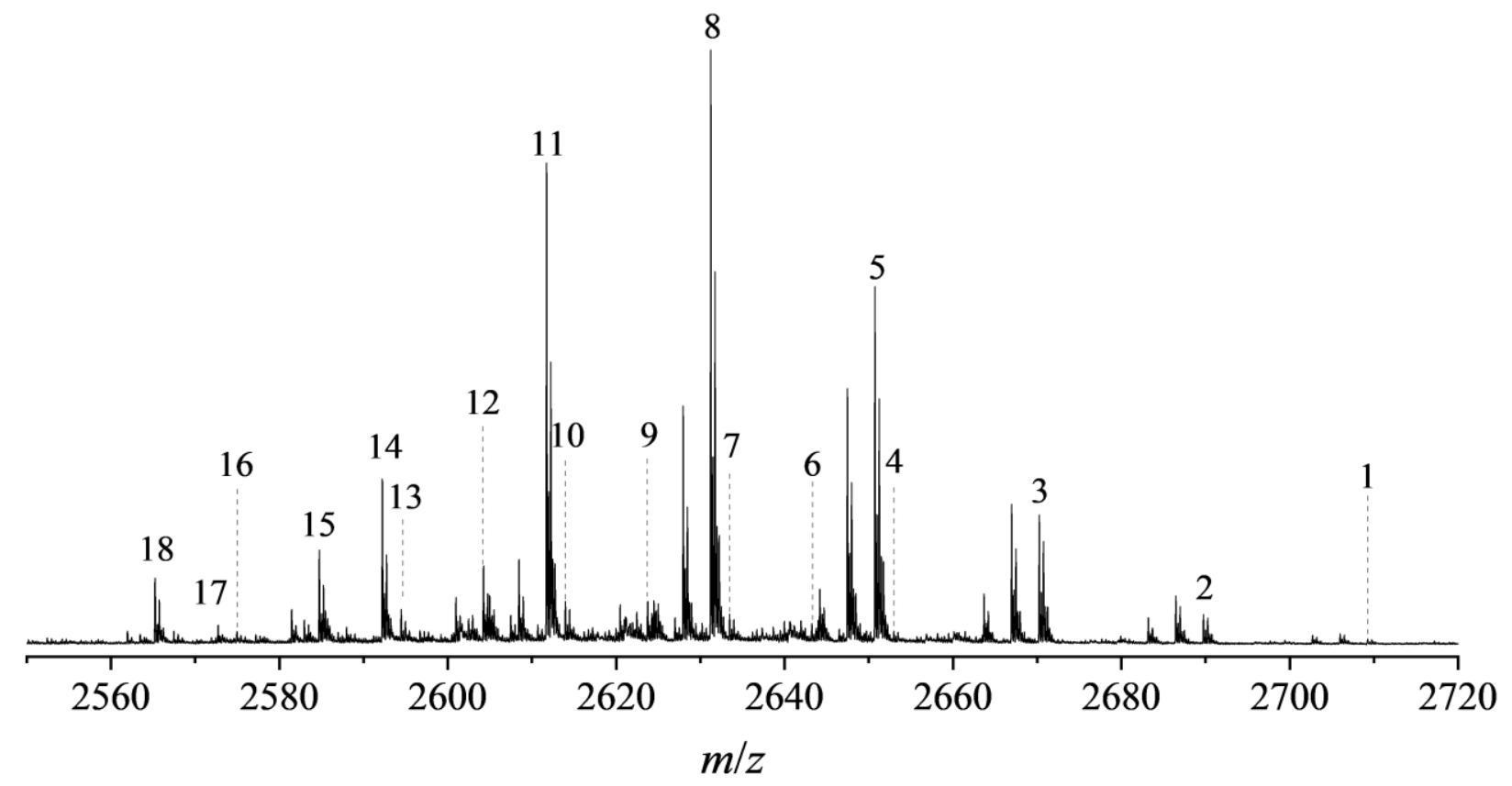

Figure S5. Cutout of ESI-MS spectrum of $\left[\mathrm{Bi}_{38} \mathrm{O}_{45}\left(\mathrm{NO}_{3}\right)_{24}(\mathrm{DMSO})_{28}\right]: \mathrm{Ce} \cdot 1.5 \mathrm{DMSO}(\mathbf{1})$ electrosprayed from a mixture of DMSO and $\mathrm{MeCN}(v: v=0.05: 0.95, c=100 \mu \mathrm{M})$ showing the quadruply positive charged ions.

Table S4. Assignment of the Quadruply Positive Charged Ions in the ESI-MS Spectrum of $\left[\mathrm{Bi}_{38} \mathrm{O}_{45}\left(\mathrm{NO}_{3}\right)_{24}(\mathrm{DMSO})_{28}\right]: \mathrm{Ce} \cdot 1.5 \mathrm{DMSO}(1)$.

\begin{tabular}{|c|c|c|c|c|c|}
\hline Label & Species & $\mathrm{M} / \mathrm{g} \cdot \mathrm{mol}^{-1}$ & $m / z$ (calc.) & $m / z(\mathrm{ESI})$ & Fragmentation \\
\hline 1 & {$\left[\mathrm{Bi}_{38} \mathrm{O}_{45}\left(\mathrm{NO}_{3}\right)_{20}(\mathrm{DMSO})_{12}\right]^{4+}$} & 10836.95 & 2709.24 & 2709.25 & - \\
\hline 2 & {$\left[\mathrm{Bi}_{38} \mathrm{O}_{45}\left(\mathrm{NO}_{3}\right)_{20}(\mathrm{DMSO})_{11}\right]^{4+}$} & 10758.93 & 2689.73 & 2689.75 & $1-$ DMSO \\
\hline 3 & {$\left[\mathrm{Bi}_{38} \mathrm{O}_{45}\left(\mathrm{NO}_{3}\right)_{20}(\mathrm{DMSO})_{10}\right]^{4+}$} & 10680.92 & 2670.23 & 2670.25 & 2 - DMSO \\
\hline 4 & {$\left[\mathrm{Bi}_{37} \mathrm{Ce}^{\mathrm{III}} \mathrm{O}_{45}\left(\mathrm{NO}_{3}\right)_{20}(\mathrm{DMSO})_{10}\right]^{4+}$} & 10611.84 & 2652.96 & 2652.97 & - \\
\hline 5 & {$\left[\mathrm{Bi}_{38} \mathrm{O}_{45}\left(\mathrm{NO}_{3}\right)_{20}(\mathrm{DMSO})_{9}\right]^{4+}$} & 10602.91 & 2650.73 & 2650.74 & 3 -DMSO \\
\hline 6 & {$\left[\mathrm{Bi}_{38} \mathrm{O}_{46}\left(\mathrm{NO}_{3}\right)_{18}(\mathrm{DMSO})_{10}\right]^{4+}$} & 10572.94 & 2643.23 & 2643.24 & $3-\mathrm{N}_{2} \mathrm{O}_{5}$ \\
\hline 7 & {$\left[\mathrm{Bi}_{37} \mathrm{Ce}^{\mathrm{III}} \mathrm{O}_{45}\left(\mathrm{NO}_{3}\right)_{20}(\mathrm{DMSO})_{9}\right]^{4+}$} & 10533.83 & 2633.46 & 2633.47 & $4-\mathrm{DMSO}$ \\
\hline 8 & {$\left[\mathrm{Bi}_{38} \mathrm{O}_{45}\left(\mathrm{NO}_{3}\right)_{20}(\mathrm{DMSO})_{8}\right]^{4+}$} & 10524.89 & 2631.22 & 2631.23 & 5 - DMSO \\
\hline 9 & {$\left[\mathrm{Bi}_{38} \mathrm{O}_{46}\left(\mathrm{NO}_{3}\right)_{18}(\mathrm{DMSO})_{9}\right]^{4+}$} & 10494.93 & 2623.73 & 2623.74 & $5-\mathrm{N}_{2} \mathrm{O}_{5}$ \\
\hline 10 & {$\left[\mathrm{Bi}_{37} \mathrm{Ce}^{\mathrm{III}} \mathrm{O}_{45}\left(\mathrm{NO}_{3}\right)_{20}(\mathrm{DMSO})_{8}\right]^{4+}$} & 10455.82 & 2613.95 & 2613.96 & $7-\mathrm{DMSO}$ \\
\hline 11 & {$\left[\mathrm{Bi}_{38} \mathrm{O}_{45}\left(\mathrm{NO}_{3}\right)_{20}(\mathrm{DMSO})_{7}\right]^{4+}$} & 10446.88 & 2611.72 & 2611.73 & $8-\mathrm{DMSO}$ \\
\hline 12 & {$\left[\mathrm{Bi}_{38} \mathrm{O}_{46}\left(\mathrm{NO}_{3}\right)_{18}(\mathrm{DMSO})_{8}\right]^{4+}$} & 10416.91 & 2604.23 & 2604.24 & $8-\mathrm{N}_{2} \mathrm{O}_{5}$ \\
\hline 13 & {$\left[\mathrm{Bi}_{37} \mathrm{Ce}^{\mathrm{III}} \mathrm{O}_{45}\left(\mathrm{NO}_{3}\right)_{20}(\mathrm{DMSO})_{7}\right]^{4+}$} & 10377.80 & 2594.45 & 2594.46 & $10-\mathrm{DMSO}$ \\
\hline 14 & {$\left[\mathrm{Bi}_{38} \mathrm{O}_{45}\left(\mathrm{NO}_{3}\right)_{20}(\mathrm{DMSO})_{6}\right]^{4+}$} & 10368.86 & 2592.22 & 2592.23 & $11-\mathrm{DMSO}$ \\
\hline 15 & {$\left[\mathrm{Bi}_{38} \mathrm{O}_{46}\left(\mathrm{NO}_{3}\right)_{18}(\mathrm{DMSO})_{7}\right]^{4+}$} & 10338.90 & 2584.72 & 2584.74 & $11-\mathrm{N}_{2} \mathrm{O}_{5}$ \\
\hline 16 & {$\left[\mathrm{Bi}_{37} \mathrm{Ce}^{\mathrm{III}} \mathrm{O}_{45}\left(\mathrm{NO}_{3}\right)_{20}(\mathrm{DMSO})_{6}\right]^{4+}$} & 10299.79 & 2574.95 & 2574.96 & $13-\mathrm{DMSO}$ \\
\hline 17 & {$\left[\mathrm{Bi}_{38} \mathrm{O}_{45}\left(\mathrm{NO}_{3}\right)_{20}(\mathrm{DMSO})_{5}\right]^{4+}$} & 10290.85 & 2572.71 & 2572.72 & $14-\mathrm{DMSO}$ \\
\hline 18 & {$\left[\mathrm{Bi}_{38} \mathrm{O}_{46}\left(\mathrm{NO}_{3}\right)_{18}(\mathrm{DMSO})_{6}\right]^{4+}$} & 10260.88 & 2565.22 & 2565.23 & $14-\mathrm{N}_{2} \mathrm{O}_{5}$ \\
\hline
\end{tabular}



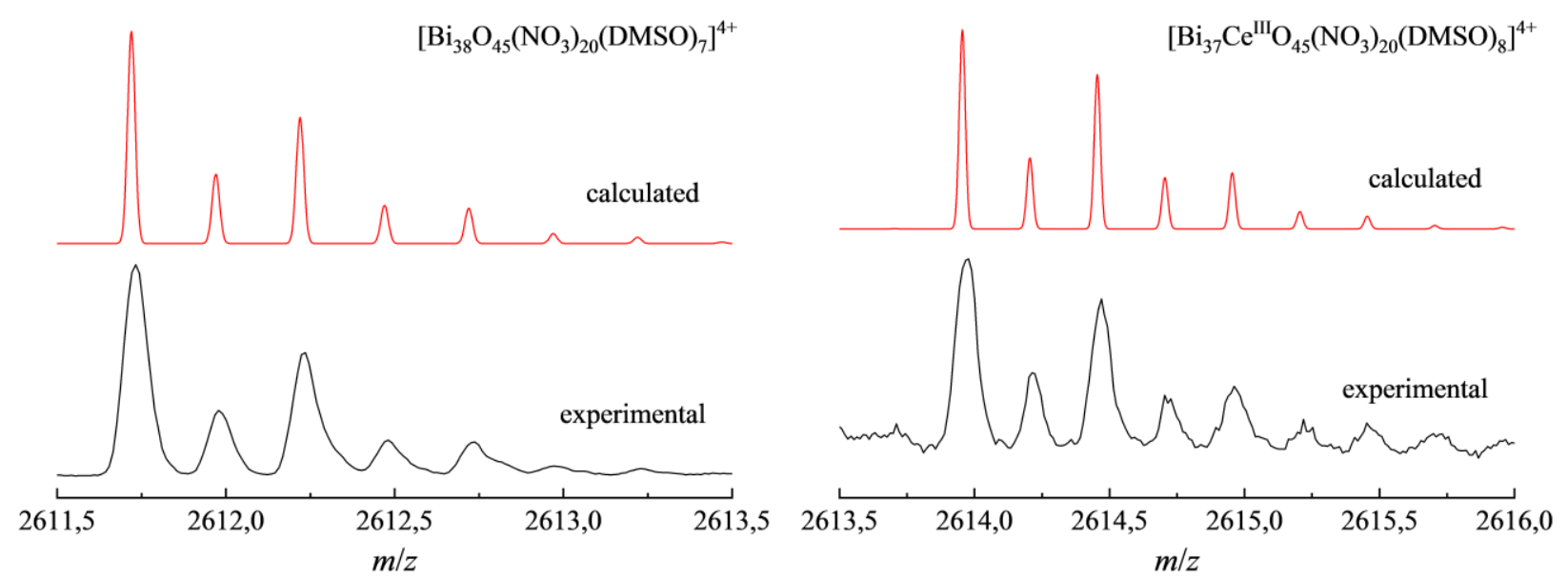

Figure S6. Calculated and observed isotope patterns of the ions $\left[\mathrm{Bi}_{38} \mathrm{O}_{45}\left(\mathrm{NO}_{3}\right)_{20}(\mathrm{DMSO})_{7}\right]^{4+}$ (left) and $\left[\mathrm{Bi}_{37} \mathrm{Ce}^{\mathrm{III}} \mathrm{O}_{45}\left(\mathrm{NO}_{3}\right)_{20}(\mathrm{DMSO})_{8}\right]^{4+}$ (right) obtained in the ESI-MS spectrum of $\left[\mathrm{Bi}_{38} \mathrm{O}_{45}\left(\mathrm{NO}_{3}\right)_{24}(\mathrm{DMSO})_{28}\right]$ :Ce--1.5DMSO (1) electrosprayed from a mixture of DMSO and $\mathrm{MeCN}(v: v=0.05: 0.95, c=100 \mu \mathrm{M})$.

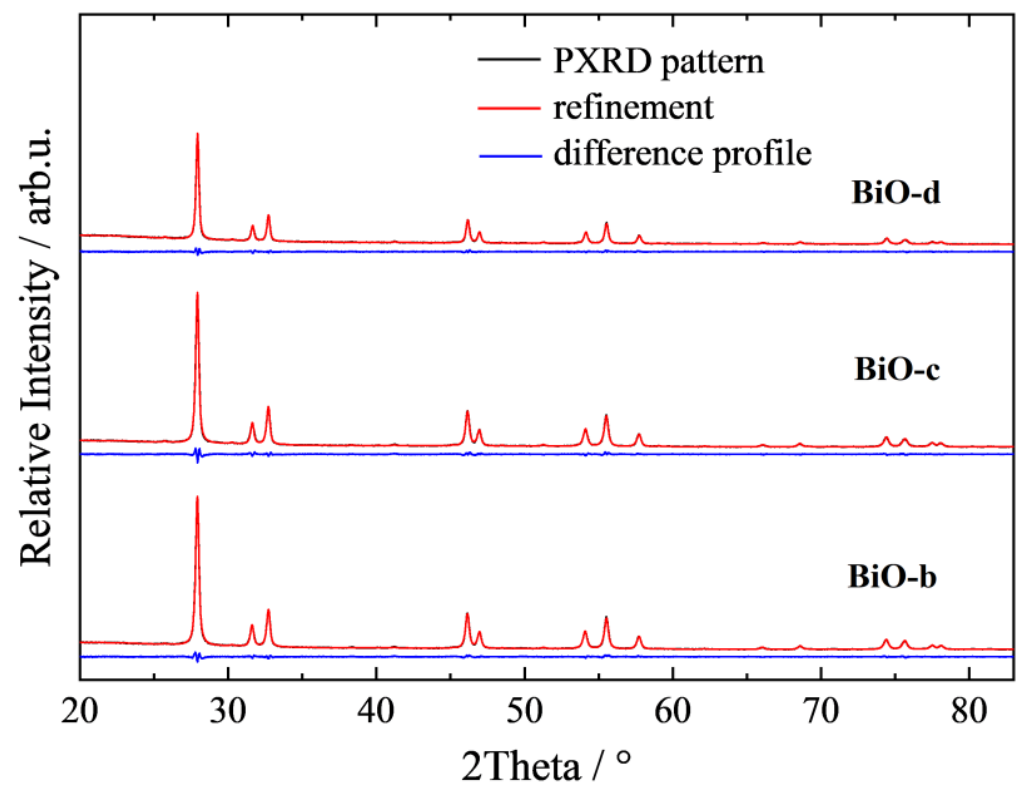

Figure S7. Refined PXRD patterns of $\beta-\mathrm{Bi}_{2} \mathrm{O}_{3}(\mathbf{B i O}-\mathbf{b}-\mathbf{B i O}-\mathbf{d})$ after thermal annealing of $\left[\mathrm{Bi}_{38} \mathrm{O}_{45}(\mathrm{OH})_{24}\right](\mathbf{B})$ in an $\mathrm{Al}_{2} \mathrm{O}_{3^{-}}$ crucible for $15 \mathrm{~min}$ at $330^{\circ} \mathrm{C}(\mathbf{b}), 370^{\circ} \mathrm{C}(\mathbf{c})$, and $400^{\circ} \mathrm{C}(\mathbf{d})$, respectively. 


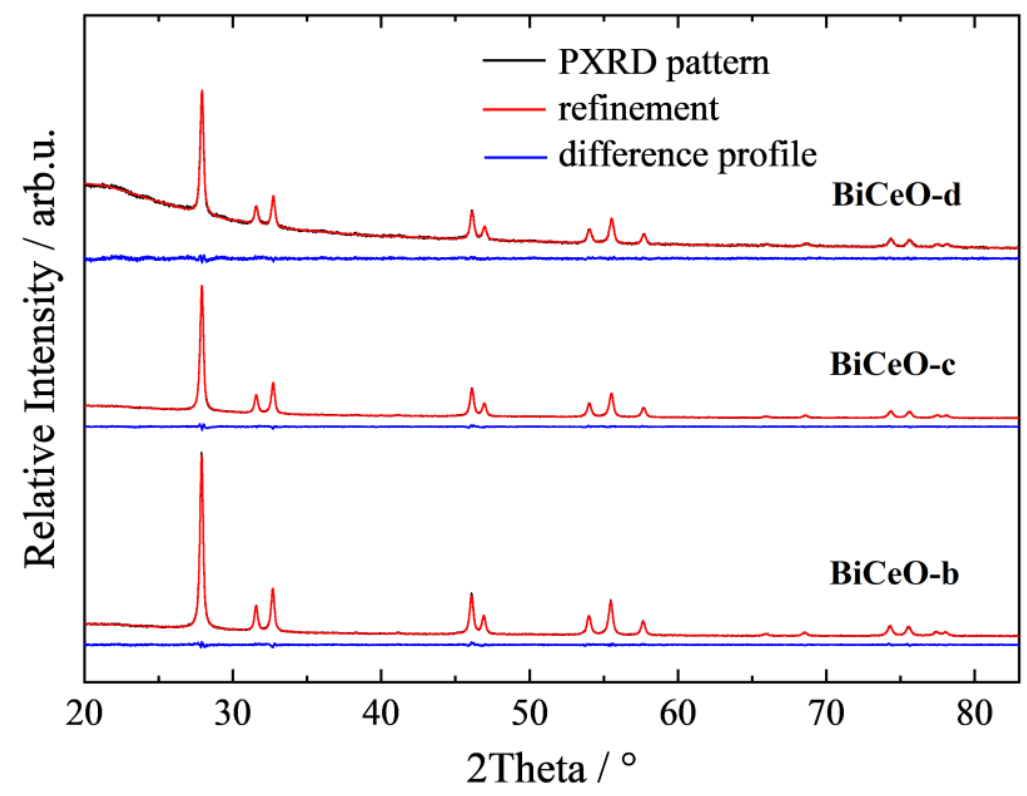

Figure S8. Refined PXRD patterns of $\beta-\mathrm{Bi}_{2} \mathrm{O}_{3}$ : $\mathrm{Ce}(\mathbf{B i C e O}-\mathbf{b}-\mathbf{B i C e O}-\mathbf{d})$ after thermal annealing of $\left[\mathrm{Bi}_{38} \mathrm{O}_{45}(\mathrm{OH})_{24}\right]$ : $\mathrm{Ce}(\mathbf{6})$ in an $\mathrm{Al}_{2} \mathrm{O}_{3}$-crucible for $15 \mathrm{~min}$ at $330^{\circ} \mathrm{C}(\mathbf{b}), 370^{\circ} \mathrm{C}(\mathbf{c})$, and $400^{\circ} \mathrm{C}(\mathbf{d})$, respectively. 
Table S5. Refined Structural Data of $\beta-\mathrm{Bi}_{2} \mathrm{O}_{3}(\mathrm{BiO}-\mathrm{a}-\mathrm{BiO}-\mathrm{d})$ and $\beta-\mathrm{Bi}_{2} \mathrm{O}_{3}: \mathrm{Ce}(\mathrm{BiCeO}-\mathrm{a}-\mathrm{BiCeO}-\mathrm{d})$ with the Space Group $P \overline{4} 2_{1}$.

\begin{tabular}{|c|c|c|c|c|c|c|c|}
\hline Sample & Atom & Site & $x$ & $y$ & $z$ & Occ. & Beq. \\
\hline \multirow[t]{3}{*}{ BiO-a } & Bi1 & $8 \mathrm{e}$ & $0.0087(3)$ & $0.2524(4)$ & $0.2527(7)$ & 1 & $2.02(3)$ \\
\hline & $\mathrm{O} 1$ & $8 \mathrm{e}$ & $0.299(3)$ & $0.297(3)$ & $0.040(6)$ & 1 & $2.02(3)$ \\
\hline & $\mathrm{O} 2$ & $4 d$ & 0.00000 & 0.50000 & $0.102(6)$ & 1 & $2.02(3)$ \\
\hline \multirow[t]{3}{*}{ BiO-b } & Bil & $8 \mathrm{e}$ & $0.01075(18)$ & $0.2534(3)$ & $0.2517(6)$ & 1 & $1.67(3)$ \\
\hline & $\mathrm{O} 1$ & $8 \mathrm{e}$ & $0.303(2)$ & $0.295(2)$ & $0.031(4)$ & 1 & $1.67(3)$ \\
\hline & $\mathrm{O} 2$ & $4 d$ & 0.00000 & 0.50000 & $0.104(4)$ & 1 & $1.67(3)$ \\
\hline \multirow[t]{3}{*}{ BiO-c } & Bil & $8 \mathrm{e}$ & $0.01302(16)$ & $0.2537(3)$ & $0.2440(5)$ & 1 & $1.36(3)$ \\
\hline & $\mathrm{O} 1$ & $8 \mathrm{e}$ & $0.3097(19)$ & $0.300(2)$ & $0.045(4)$ & 1 & $1.36(3)$ \\
\hline & $\mathrm{O} 2$ & $4 \mathrm{~d}$ & 0.00000 & 0.50000 & $0.076(5)$ & 1 & $1.36(3)$ \\
\hline \multirow[t]{3}{*}{ BiO-d } & Bil & $8 \mathrm{e}$ & $0.0134(2)$ & $0.2522(5)$ & $0.2570(5)$ & 1 & $1.91(3)$ \\
\hline & $\mathrm{O} 1$ & $8 \mathrm{e}$ & $0.294(2)$ & $0.3069(19)$ & $0.056(5)$ & 1 & $1.91(3)$ \\
\hline & $\mathrm{O} 2$ & $4 d$ & 0.00000 & 0.50000 & $0.079(7)$ & 1 & $1.91(3)$ \\
\hline \multirow[t]{5}{*}{ BiCeO-a } & Bil & $8 \mathrm{e}$ & $-0.0021(9)$ & $0.2537(3)$ & $0.2583(4)$ & 0.985 & $2.59(2)$ \\
\hline & Cel & $8 \mathrm{e}$ & $-0.0021(9)$ & $0.2537(3)$ & $0.2583(4)$ & 0.015 & $2.59(2)$ \\
\hline & $\mathrm{O} 1$ & $8 \mathrm{e}$ & $0.208(4)$ & $0.195(4)$ & $0.040(7)$ & 1 & $2.59(2)$ \\
\hline & $\mathrm{O} 2$ & $4 d$ & 0.00000 & 0.50000 & $0.100(7)$ & 1 & $2.59(2)$ \\
\hline & $\mathrm{O} 3$ & $2 b$ & 0.00000 & 0.00000 & 0.50000 & 0.03 & $2.59(2)$ \\
\hline \multirow[t]{5}{*}{ BiCeO-b } & Bil & $8 \mathrm{e}$ & $-0.0056(4)$ & $0.2537(4)$ & $0.2582(4)$ & 0.985 & $1.95(3)$ \\
\hline & $\mathrm{Ce} 1$ & $8 \mathrm{e}$ & $-0.0056(4)$ & $0.2537(4)$ & $0.2582(4)$ & 0.015 & $1.95(3)$ \\
\hline & $\mathrm{O} 1$ & $8 \mathrm{e}$ & $0.201(3)$ & $0.198(3)$ & $0.040(6)$ & 1 & $1.95(3)$ \\
\hline & $\mathrm{O} 2$ & $4 d$ & 0.00000 & 0.50000 & $0.099(6)$ & 1 & $1.95(3)$ \\
\hline & $\mathrm{O} 3$ & $2 b$ & 0.00000 & 0.00000 & 0.50000 & 0.03 & $1.95(3)$ \\
\hline \multirow[t]{5}{*}{ BiCeO-c } & Bil & $8 \mathrm{e}$ & $-0.0068(3)$ & $0.2532(4)$ & $0.2570(5)$ & 0.985 & $2.22(3)$ \\
\hline & Cel & $8 \mathrm{e}$ & $-0.0068(3)$ & $0.2532(4)$ & $0.2570(5)$ & 0.015 & $2.22(3)$ \\
\hline & $\mathrm{O} 1$ & $8 \mathrm{e}$ & $0.206(3)$ & $0.192(3)$ & $0.058(6)$ & 1 & $2.22(3)$ \\
\hline & $\mathrm{O} 2$ & $4 d$ & 0.00000 & 0.50000 & $0.083(8)$ & 1 & $2.22(3)$ \\
\hline & $\mathrm{O} 3$ & $2 b$ & 0.00000 & 0.00000 & 0.50000 & 0.03 & $2.22(3)$ \\
\hline \multirow[t]{5}{*}{ BiCeO-d } & Bil & $8 \mathrm{e}$ & $-0.0062(10)$ & $0.2448(9)$ & $0.2586(11)$ & 0.985 & $1.35(8)$ \\
\hline & $\mathrm{Ce} 1$ & $8 \mathrm{e}$ & $-0.0062(10)$ & $0.2448(9)$ & $0.2586(11)$ & 0.015 & $1.35(8)$ \\
\hline & $\mathrm{O} 1$ & $8 \mathrm{e}$ & $0.208(7)$ & $0.197(7)$ & $0.066(13)$ & 1 & $1.35(8)$ \\
\hline & $\mathrm{O} 2$ & $4 d$ & 0.00000 & 0.50000 & $0.077(17)$ & 1 & $1.35(8)$ \\
\hline & $\mathrm{O} 3$ & $2 b$ & 0.00000 & 0.00000 & 0.50000 & 0.03 & $1.35(8)$ \\
\hline
\end{tabular}




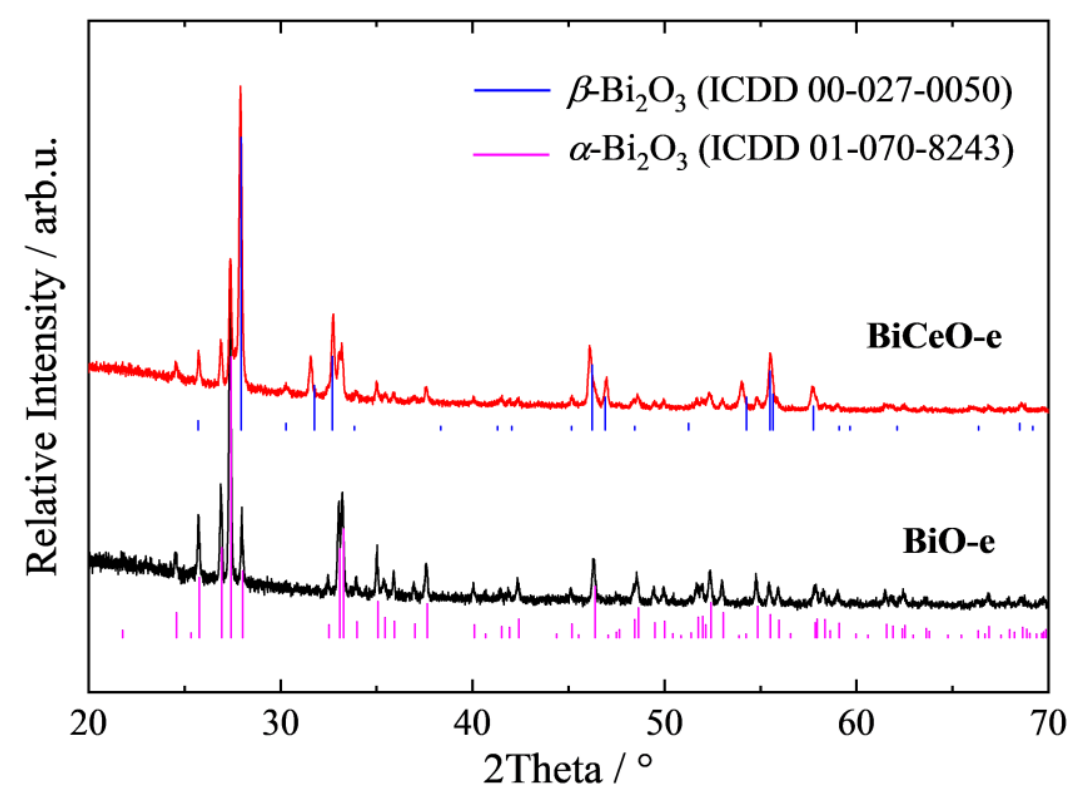

Figure 59. PXRD patterns of $\alpha-\mathrm{Bi}_{2} \mathrm{O}_{3}(\mathbf{B i O}-\mathbf{e})$ and $\alpha-/ \beta-\mathrm{Bi}_{2} \mathrm{O}_{3}: \mathrm{Ce}(\mathbf{B i C e O}-\mathbf{e})$ after calcination $\left(\dot{V}_{\text {air }}=50 \mathrm{~L} \cdot \mathrm{h}^{-1}\right)$ of $\left[\mathrm{Bi}_{38} \mathrm{O}_{45}(\mathrm{OH})_{24}\right](\mathbf{B})$ and $\left[\mathrm{Bi}_{38} \mathrm{O}_{45}(\mathrm{OH})_{24}\right]: \mathrm{Ce}(\mathbf{6})$ at $T=600{ }^{\circ} \mathrm{C}$ on silver sheets for $60 \mathrm{~min}$.

Table S6. Refined Cell Parameters of $\beta-\mathrm{Bi}_{2} \mathrm{O}_{3}(\mathrm{BiO}-\mathrm{a}-\mathrm{BiO}-\mathrm{d})$ and $\beta-\mathrm{Bi}_{2} \mathrm{O}_{3}: \mathrm{Ce}(\mathrm{BiCeO}-\mathrm{a}-\mathrm{BiCeO}-\mathrm{d})$ with the Space Group $P \overline{\mathbf{4}} \mathbf{2}_{1}$.

\begin{tabular}{ccccccc}
\hline Sample & $a / \AA$ & $c / \AA$ & $V / \AA^{3}$ & $d_{p} / \mathrm{nm}$ & $D_{x} / \mathrm{g} \cdot \mathrm{cm}^{-3}$ & $R_{\text {wp }} / \%$ \\
\hline BiO-a & $7.72755(14)$ & $5.65405(12)$ & $337.632(15)$ & $46.3(5)$ & $9.1667(4)$ & 2.69 \\
BiO-b & $7.72664(11)$ & $5.64934(9)$ & $337.270(11)$ & $49.6(4)$ & $9.1765(3)$ & 3.40 \\
BiO-c & $7.72813(12)$ & $5.64605(10)$ & $337.205(12)$ & $58.0(7)$ & $9.1783(3)$ & 3.99 \\
BiO-d & $7.72770(13)$ & $5.64361(11)$ & $337.021(13)$ & $71.6(11)$ & $9.1833(4)$ & 3.17 \\
BiCeO-a & $7.73478(12)$ & $5.66294(11)$ & $338.795(12)$ & $52.4(3)$ & $9.0994(3)$ & 3.97 \\
BiCeO-b & $7.73312(13)$ & $5.65753(11)$ & $338.327(13)$ & $57.3(7)$ & $9.1120(4)$ & 3.19 \\
BiCeO-c & $7.72693(12)$ & $5.65443(11)$ & $337.600(12)$ & $56.4(7)$ & $9.1316(3)$ & 2.60 \\
BiCeO-d & $7.7242(3)$ & $5.6548(2)$ & $337.38(3)$ & $59.4(17)$ & $9.1375(7)$ & 1.83 \\
\hline
\end{tabular}

Table S7. Refined Bond Distances in $\beta$ - $\mathrm{Bi}_{2} \mathrm{O}_{3}(\mathrm{BiO}-\mathrm{a}-\mathrm{BiO}-\mathrm{d})$ and $\beta-\mathrm{Bi}_{2} \mathrm{O}_{3}: \mathrm{Ce}(\mathrm{BiCeO}-\mathrm{a}-\mathrm{BiCeO}-\mathrm{d})$ with the Space Group $P \overline{\mathbf{4}} \mathbf{2}_{1}$ c.

\begin{tabular}{cccc}
\hline Sample & $d(\mathrm{Bi} 1 / \mathrm{Ce} 1-\mathrm{O} 1) / \AA$ & $d(\mathrm{Bi} 1 / \mathrm{Ce} 1-\mathrm{O} 2) / \AA$ & $d(\mathrm{Bi} 1 / \mathrm{Ce} 1-\mathrm{O} 3) / \AA$ \\
\hline BiO-a & $2.03(3)-2.91(3)$ & $2.098(14)-2.75(3)$ & - \\
BiO-b & $2.055(19)-2.88(2)$ & $2.083(9)-2.756(17)$ & - \\
BiO-c & $2.014(18)-2.952(18)$ & $2.128(12)-2.67(2)$ & - \\
BiO-d & $2.05(2)-3.06(2)$ & $2.165(18)-2.64(3)$ & $2.393(3)$ \\
BiCeO-a & $2.09(3)-2.88(3)$ & $2.106(16)-2.71(3)$ & $2.392(3)$ \\
BiCeO-b & $2.06(3)-2.89(3)$ & $2.108(15)-2.71(3)$ & $2.391(3)$ \\
BiCeO-c & $2.05(3)-2.99(2)$ & $2.147(19)-2.65(3)$ & $2.332(7)$ \\
BiCeO-d & $2.01(6)-2.98(6)$ & $2.22(4)-2.67(6)$ & \\
\hline
\end{tabular}




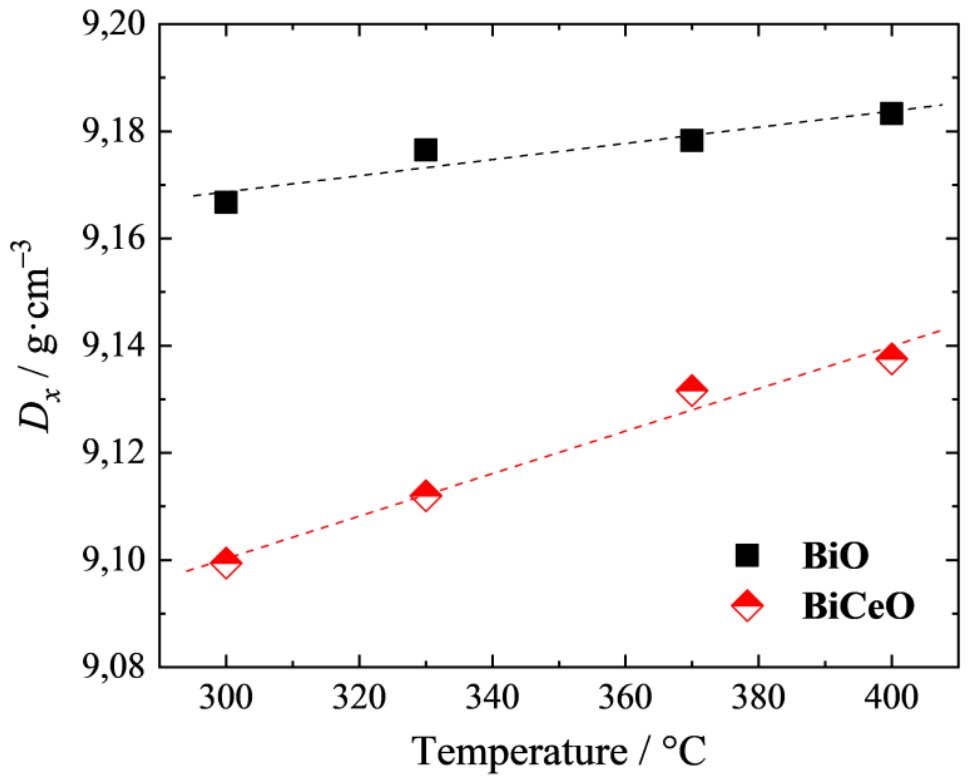

Figure S10. Increase of the density $D_{x}$ based on the Rietveld refinement of $\beta-\mathrm{Bi}_{2} \mathrm{O}_{3}(\mathbf{B i O})$ and $\beta-\mathrm{Bi}_{2} \mathrm{O}_{3}: \mathrm{Ce}(\mathbf{B i C e O})$ obtained after the annealing of $\left[\mathrm{Bi}_{38} \mathrm{O}_{45}(\mathrm{OH})_{24}\right](\mathbf{B})$ and $\left[\mathrm{Bi}_{38} \mathrm{O}_{45}(\mathrm{OH})_{24}\right]: \mathrm{Ce}(\mathbf{6})$ in the temperature range of $300-400{ }^{\circ} \mathrm{C}$.

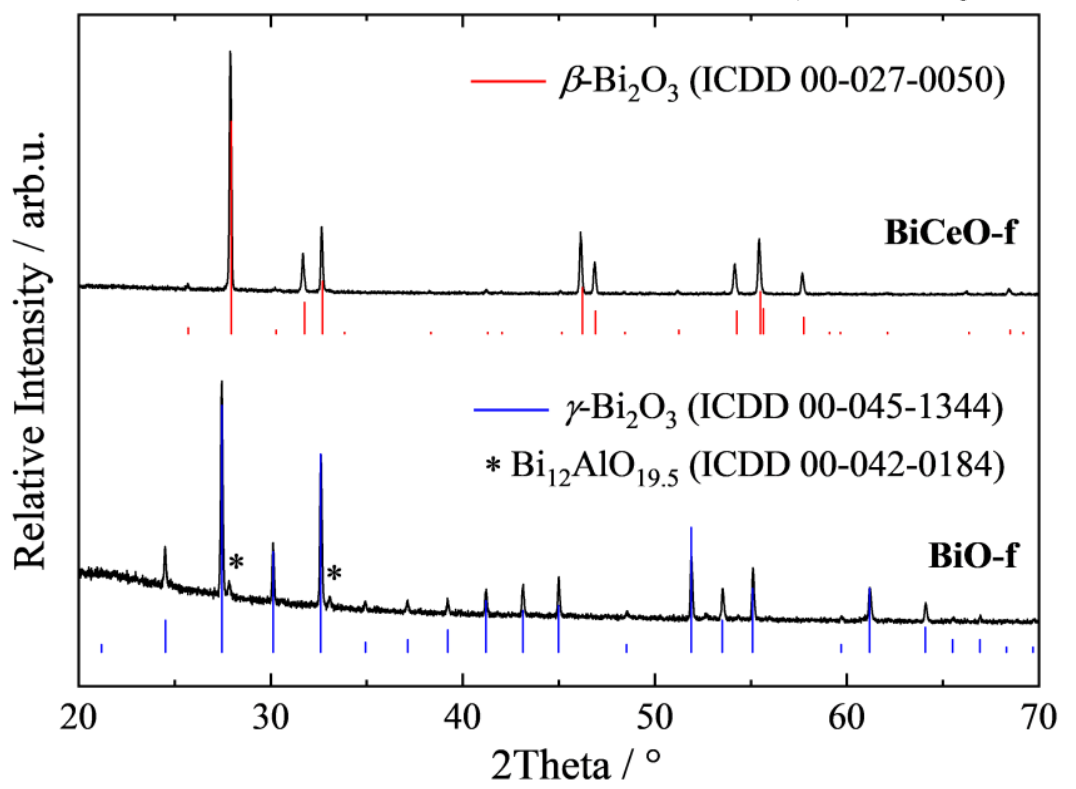

Figure S11. PXRD patterns of $\beta-\mathrm{Bi}_{2} \mathrm{O}_{3}: \mathrm{Ce}$ (BiCeO-f) and $\gamma-\mathrm{Bi}_{2} \mathrm{O}_{3} / \mathrm{Bi}_{12} \mathrm{AlO}_{19.5}$ (BiO-f) after DSC analysis of $\beta-\mathrm{Bi}_{2} \mathrm{O}_{3}: \mathrm{Ce}$ (BiCeO-a) and $\beta-\mathrm{Bi}_{2} \mathrm{O}_{3}\left(\mathbf{B i O}-\mathbf{a}\right.$ ) in the temperature range of $T=40-800{ }^{\circ} \mathrm{C}$ (in an $\mathrm{Al}_{2} \mathrm{O}_{3}$ crucible, held for $60 \mathrm{~min}$ ), followed by subsequent cooling.

Table S8. Results of the Photocatalytic Decomposition of Aqueous Triclosan $(c=40 \mu \mathrm{M})$ in the Presence of $\beta-\mathrm{Bi}_{2} \mathrm{O}_{3}$ $(\mathrm{BiO}-\mathrm{a}, \mathrm{BiO}-\mathrm{c})$ and $\beta-\mathrm{Bi}_{2} \mathrm{O}_{3}: \mathrm{Ce}(\mathrm{BiCeO}-\mathrm{a}, \mathrm{BiCeO}-\mathrm{c})$ Under Visible-Light Irradiation $(420 \mathrm{~nm} \leq \lambda \leq 700 \mathrm{~nm})$. 


\begin{tabular}{|c|c|c|c|c|c|c|c|}
\hline Sample & $E_{g} / \mathrm{eV}$ & $A_{\mathrm{BET}} / \mathrm{m}^{2} \cdot \mathrm{g}^{-1}$ & {$[\mathrm{TCS}]_{0} / \%$} & {$[\mathrm{TCS}]_{150} / \%$} & $k / \mathrm{s}^{-1}$ & $k / \min ^{-1}$ & $m_{\text {residue }} / \mathrm{mg}$ \\
\hline $\begin{array}{c}\text { Photolysis } \\
\text { TCS }\end{array}$ & - & - & 100 & 92 & $4.60 \cdot 10^{-6}$ & $3.31 \cdot 10^{-4}$ & 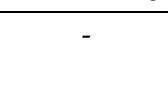 \\
\hline BiO-a & 2.38 & 24 & 86 & 9 & $\begin{array}{l}4.96 \cdot 10^{-4}, \\
4.96 \cdot 10^{-5}\end{array}$ & $\begin{array}{l}0.033 \\
0.003\end{array}$ & 7 \\
\hline BiO-c & 2.36 & 20 & 95 & 8 & $\begin{array}{l}5.24 \cdot 10^{-4}, \\
1.40 \cdot 10^{-4}\end{array}$ & $\begin{array}{l}0.035 \\
0.009\end{array}$ & 7 \\
\hline BiCeO-a & 2.29 & 23 & 81 & 6 & $\begin{array}{l}4.31 \cdot 10^{-4} \\
2.25 \cdot 10^{-4}\end{array}$ & $\begin{array}{l}0.026 \\
0.013\end{array}$ & 6 \\
\hline BiCeO-c & 2.27 & 1 & 98 & 78 & $2.54 \cdot 10^{-5}$ & 0.002 & 7 \\
\hline
\end{tabular}

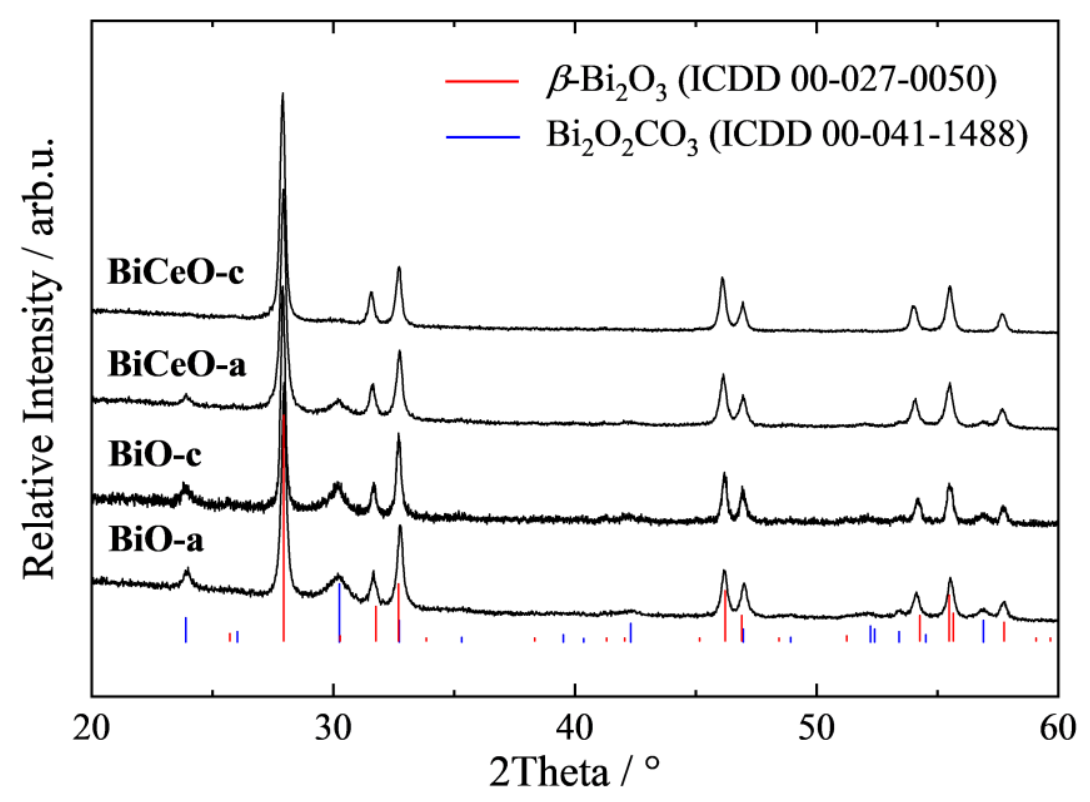

Figure S12. PXRD patterns of $\beta-\mathrm{Bi}_{2} \mathrm{O}_{3}(\mathbf{B i O}-\mathbf{a}, \mathbf{B i O}-\mathbf{c})$ and $\beta-\mathrm{Bi}_{2} \mathrm{O}_{3}: \mathrm{Ce}(\mathbf{B i C e O}-\mathbf{a}, \mathbf{B i C e O}-\mathbf{c})$ after photocatalytic decomposition of triclosan $(c=40 \mu \mathrm{M})$ under visible-light irradiation. 


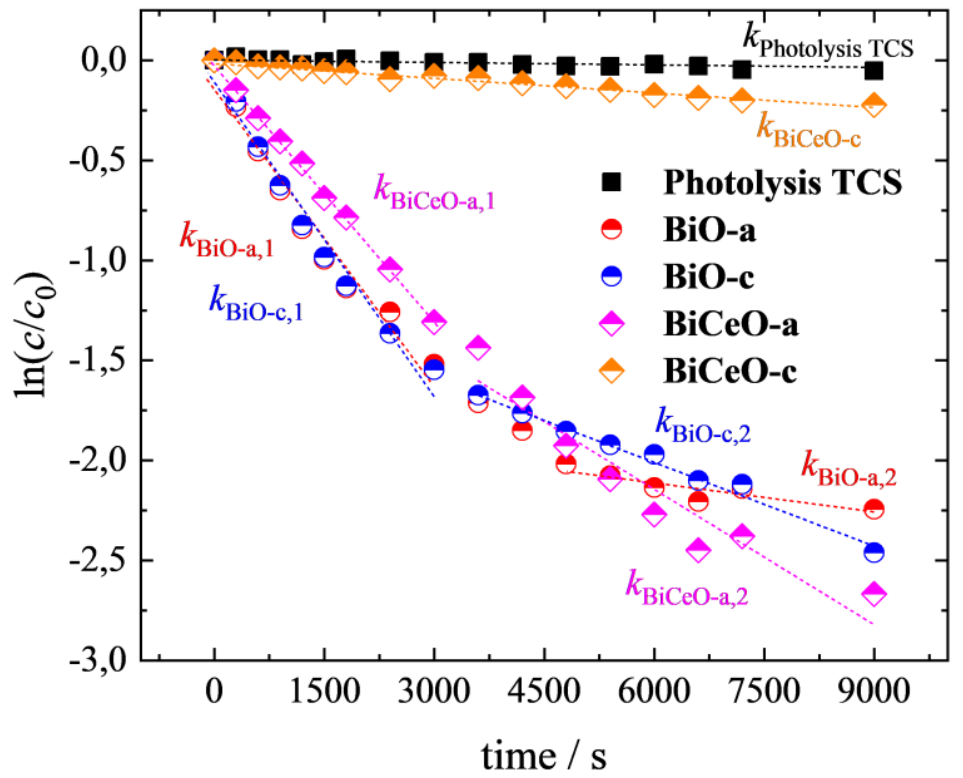

Figure S13. Semilogarithmic plots of the photodegradation of aqueous triclosan $(c=40 \mu \mathrm{M})$ under visible-light irradiation $(420 \mathrm{~nm} \leq \lambda \leq 700 \mathrm{~nm})$ in the presence of $\beta-\mathrm{Bi}_{2} \mathrm{O}_{3}(\mathbf{B i O}-\mathbf{a}, \mathbf{B i O}-\mathbf{c})$ and $\beta-\mathrm{Bi}_{2} \mathrm{O}_{3}: \mathrm{Ce}(\mathbf{B i C e O}-\mathbf{a}, \mathbf{B i C e O}-\mathbf{c})$.

\section{REFERENCES:}

1. Miersch, L.; Schlesinger, M.; Troff, R. W.; Schalley, C. A.; Rüffer, T.; Lang, H.; Zahn, D.; Mehring, M. Hydrolysis of a Basic Bismuth Nitrate-Formation and Stability of Novel Bismuth Oxido Clusters. Chem.- Eur. J. 2011, 17, 69856990.

2. $\quad$ Moulder, J. F.; Stickle, W. F.; Sobol, P. E.; Bomben, K. D. Handbook of X-ray Photoelectron Spectroscopy; PerkinElmer Corp.: Eden Prairie, MN, 1992.

3. Beche, E.; Charvin, P.; Perarnau, D.; Abanades, S.; Flamant, G. Ce 3d XPS Investigation of Cerium Oxides and Mixed Cerium Oxide $\left(\mathrm{Ce}_{x} \mathrm{Ti}_{y} \mathrm{O}_{z}\right)$. Surf. Interface Anal. 2008, 40, 264-267.

4. Beche, E.; Peraudeau, G.; Flaud, V.; Perarnau, D. An XPS Investigation of $\left(\mathrm{La}_{2} \mathrm{O}_{3}\right)_{1-x}\left(\mathrm{CeO}_{2}\right)_{2 x}\left(\mathrm{ZrO}_{2}\right)_{2} \mathrm{Compounds}_{\text {. }}$ Surf. Interface Anal. 2012, 44, 1045-1050. 